\title{
Analysis of Clinical Characteristics of 556 Spinal Tuberculosis Patients in Two Tertiary Teaching Hospitals in Guangxi Province
}

\author{
Hao Zeng, ${ }^{1,2}$ Yingfang Liang, ${ }^{3}$ Jiaguo He, ${ }^{4}$ Liyi Chen, ${ }^{1}$ Haixia Su, ${ }^{5}$ Simi Liao, ${ }^{6}$ \\ Sichun Huang, ${ }^{1}$ and Hongyu Qin (1) ${ }^{1,2}$ \\ ${ }^{1}$ Department of Spine and Osteopathy Surgery, Guangxi Medical University First Affiliated Hospital, 6\#Shuangyong Rd, Nanning, \\ 530000 Guangxi, China \\ ${ }^{2}$ Guangxi Key Laboratory of Regenerative Medicine, Guangxi Medical University, 22\#Shuangyong Rd, Nanning, \\ 530000 Guangxi, China \\ ${ }^{3}$ Department of Comprehensive Internal Medicine, Guangxi Medical University Affiliated Tumor Hospital, 71\#Hedi Rd, Nanning, \\ 530000 Guangxi, China \\ ${ }^{4}$ Department of Rehabilitation Medicine, Guangxi Medical University First Affiliated Hospital, 6\#Shuangyong Rd, Nanning, \\ 530000 Guangxi, China \\ ${ }^{5}$ Department of Statistics, School of Public Health, Guangxi Medical University, 22\#Shuangyong Rd, Nanning, \\ 530000 Guangxi, China \\ ${ }^{6}$ Department of Nutrition and Food Hygiene, School of Public Health, Guangxi Medical University, 22\#Shuangyong Rd, Nanning, \\ 530000 Guangxi, China
}

Correspondence should be addressed to Hongyu Qin; 414135872@qq.com

Hao Zeng and Yingfang Liang contributed equally to this work.

Received 2 September 2021; Accepted 17 November 2021; Published 10 December 2021

Academic Editor: Panagiotis Korovessis

Copyright (c) 2021 Hao Zeng et al. This is an open access article distributed under the Creative Commons Attribution License, which permits unrestricted use, distribution, and reproduction in any medium, provided the original work is properly cited.

Spinal tuberculosis (STB), which accounts for half of musculoskeletal tuberculosis, is among the leading causes of extrapulmonary tuberculosis. Guangxi Province, located in southern China, is among the most severely affected provinces in China. In this study, we collected and analyzed data from 2 Class-A tertiary teaching hospitals in Nanning City, Guangxi Province, from 2011 to 2019, with the aim of providing reference points for the prevention, diagnosis, treatment, and prognosis analysis of STB, using the epidemiological characteristics of 556 STB cases. Our results revealed that males had a slightly higher incidence $(50.17 \%)$ compared to females $(49.83 \%)$, with $64.93 \%$ of cases falling between the ages of 18 and 45 years. Cases from rural communities accounted for $63.49 \%$ of the reviewed cases. The average time between onset of symptoms and hospitalization was 18.0 months (range: 1 day-220 months). The most commonly reported symptoms were lower back pain (78.60\%), radicular pain $(51.98 \%)$, and systemic toxemia (43.53\%). Additionally, 53.98\% of the reviewed cases had varying degrees of neurological impairment. The main pathological lesion locations were the lumbar spine (43.53\%) and thoracic spine (32.55\%). Among them, $72.66 \%$ of cases involved at least 2 vertebral segments, and $62.77 \%$ of cases presented with paravertebral abscesses. Among the cases reviewed, 90.65\% underwent antituberculosis chemotherapy prior to surgery. Following treatment, the cure rate was $78.41 \%$, while $3.78 \%$ of patients had postoperative relapse. There were cases of concomitant illnesses among the cases reviewed, $40.65 \%$ of patients also had pulmonary tuberculosis, $15.29 \%$ had hepatitis B, $13.30 \%$ had diabetes, and $7.91 \%$ had hypertension. Our results still demonstrate that spinal tuberculosis remains a serious public health problem in Guangxi Province. Thus, preventive measures should be directed towards rural residents with comorbidities such as the elderly and diabetic. 


\section{Introduction}

Tuberculosis (TB) continues to be an infectious disease nightmare in developing countries. Late treatment of pulmonary tuberculosis still continues to pose a serious threat to public health and socioeconomic development in the developing world as it leads to incapacitation and uncontrolled spread [1]. Spinal tuberculosis (STB), which accounts for approximately $50 \%$ of musculoskeletal tuberculosis, is the most common extrapulmonary tuberculosis [2]. The diagnosis of spinal tuberculosis is relatively difficult. Pathological evidence from tissue samples is the gold standard for diagnosis, but in fact, it is very difficult to identify Mycobacterium tuberculosis from the lesion [2].

In addition, because of the vague nature of the presenting symptoms and paucity of neurological symptoms and signs in the early stages of the disease, most patients report serious manifestations like irreversible neurological impairments such as paralysis at the time of reporting, which increases the length of stay and treatment costs, resulting in serious financial burden [3].

Antituberculous chemotherapy remains the fundamental treatment modality for STB, with surgery as an adjuvant $[4,5]$. According to the World Health Organization report, although the incidence and mortality rates of tuberculosis (TB) in China have been declining steadily in recent years, China still remains one of the countries with the highest TB burden in the world [6]. There were approximately 833,000 new tuberculosis cases in China in 2019 , with a reported 388,000 mortalities, demonstrating that tuberculosis is still a serious public health problem among Chinese people [6].

The epidemiology of tuberculosis is influenced by factors such as geographic location, lifestyle, socioeconomic development, and the availability of good medical services. Guangxi Province, located in southern China, has a population of over 50.12 million, with a rural population of 33.22 million according to 2021 population data. Guangxi Province is among the provinces with the highest tuberculosis burden in China, with a 2019 annual incidence of 81.12/ 100,000 compared to the national incidence of 44.45 / 100,000. This therefore necessitates more studies into the prevention and treatment measures of tuberculosis-related diseases in the province. There are currently few epidemiological investigations into spinal tuberculosis in Guangxi; thus, this study investigates this common extrapulmonary disease in order to better understand the incidence, as well as provide references for prevention and treatment in the province and beyond. This study collected and analyzed inpatient data such as clinical symptoms, diagnosis, treatment, and prognosis of 556 confirmed spinal TB cases from 2 tertiary teaching hospitals in Nanning City, in order to investigate the stated goals.

\section{Materials and Methods}

2.1. Patient Data. We retrospectively analyzed the medical records of STB patients admitted to 2 tertiary teaching hospitals from January 2011 to December 2019 using the medical record retrieval code. The collected data included clinical features such as pain, radiculopathy, systemic symptoms, neurological manifestations, imaging features, pathologic vertebrae, treatment options, and posttreatment complications. Other clinical data such as comorbidities, as well as prognostic indicators, were also analyzed. Personal information such as gender, age, residence (rural or urban), and occupation was also analyzed. The Frankel grading system was used to assess the grades of the neurological manifestations in the reviewed patients; grade A: complete neurological deficit; grade B: sparing of sensation; grade C: sparing of sensation but no useful motor function; grade D: sparing of sensation and useful motor function; and grade E: no deficits. Since pulmonary tuberculosis (PTB) and diabetes are common comorbidities of STB, we analyzed the correlation between PTB, diabetes, and STB. Guangxi Province also has a high incidence of hepatitis B and hypertension; hence, their correlations were also analyzed.

2.2. Diagnosis and Treatment. The diagnostic criteria for STB are as follows: (1) clinical signs and symptoms, such as back pain, spinal deformity, neurological deficits (numbness, weakness, hypoesthesia, dyskinesia, and paralysis), and systemic toxemic symptoms (low-grade fever, night sweats), and weight loss; (2) CT and MRI findings: vertebral destruction, intervertebral space involvement, kyphosis, paravertebral abscess, etc.; (3) laboratory findings: anemia, elevated erythrocyte sedimentation rate (ESR) and Creactive protein (CRP), positive T-SPOT, tuberculosis antibodies (IgG and IgM), and positive tuberculin skin test; (4) diagnostic antituberculosis chemotherapy which was effective in difficult to diagnose cases; and (5) pathological evidence from tissue samples which is the gold standard for diagnosis.

All patients received the combination of the 4 antituberculosis chemotherapeutic agents HREZ (rifampicin $(450 \mathrm{mg}$ / day), isoniazid (300 mg/day), ethambutol (750 mg/day), and pyrazinamide $(750 \mathrm{mg} /$ day $))$ for at least 18 months. All patients underwent rigorous monitoring of renal and liver functions during treatment. All patients and their immediate families were also educated and trained to understand the importance of early, regular, and full completion of the combined medication, and family members were urged to help patients adhere to their medications. Patients were also advised to rest and avoid strenuous activities. None of the patients included in the study were HIV positive.

Patients were evaluated by telephone follow-up and outpatient visitation to gather information on posttreatment prognosis. Patients were classified as "cured" if there is no recurrent TB lesion within 2 years after treatment, the ESR level remained within the normal range, bone healing is observed on follow-up imaging examinations, and the clinical symptoms have disappeared for at least 3 months [7]. Relapses were presented as postoperative recurrence of TB lesions, sinus formation, progressive kyphosis, abscess formation, or internal fixation fractures. Patients were classified as "other" because of incomplete follow-up data, loss of contact, failure to heal, transferred treatment, or death [8]. 
TABLE 1: Demographics and epidemiology of inpatients with STB.

\begin{tabular}{|c|c|c|c|c|c|c|c|c|c|c|c|c|c|}
\hline \multirow{2}{*}{ Year } & \multicolumn{4}{|c|}{ Age (years) } & \multicolumn{2}{|c|}{ Gender } & \multicolumn{2}{|c|}{ Residence } & \multicolumn{4}{|c|}{ Occupation } & \multirow{2}{*}{ Tota } \\
\hline & $<18$ & $18 \sim 45$ & $46 \sim 60$ & $>60$ & Male & Female & Rural & Urban & Farmer & Worker & Student & Others & \\
\hline 2011 & 3 & 29 & 18 & 10 & 31 & 29 & 40 & 20 & 28 & 13 & 2 & 17 & 60 \\
\hline 2012 & 3 & 25 & 8 & 4 & 21 & 19 & 26 & 14 & 22 & 5 & 1 & 12 & 40 \\
\hline 2013 & 2 & 21 & 10 & 6 & 23 & 16 & 23 & 16 & 19 & 6 & 2 & 12 & 39 \\
\hline 2014 & 2 & 17 & 7 & 4 & 17 & 13 & 17 & 13 & 15 & 4 & 1 & 10 & 30 \\
\hline 2015 & 3 & 24 & 6 & 5 & 25 & 13 & 25 & 13 & 20 & 5 & 2 & 11 & 38 \\
\hline 2016 & 7 & 51 & 11 & 7 & 31 & 45 & 45 & 31 & 55 & 10 & 5 & 6 & 76 \\
\hline 2017 & 9 & 77 & 15 & 11 & 54 & 58 & 78 & 34 & 75 & 20 & 5 & 12 & 112 \\
\hline 2018 & 6 & 59 & 12 & 8 & 36 & 49 & 50 & 35 & 54 & 12 & 6 & 13 & 85 \\
\hline 2019 & 4 & 58 & 11 & 3 & 41 & 35 & 49 & 27 & 50 & 14 & 2 & 10 & 76 \\
\hline Total & 39 & 361 & 98 & 58 & 279 & 277 & 353 & 203 & 338 & 89 & 26 & 103 & 556 \\
\hline Rate (\%) & 7.01 & 64.93 & 17.63 & 10.43 & 50.17 & 49.83 & 63.48 & 36.51 & 60.79 & 16.01 & 4.68 & 18.53 & \\
\hline$\chi^{2}$ & \multicolumn{4}{|c|}{46.929} & \multicolumn{2}{|c|}{10.923} & \multicolumn{2}{|c|}{4.593} & \multicolumn{4}{|c|}{37.833} & \\
\hline$p$ & \multicolumn{4}{|c|}{0.003} & \multicolumn{2}{|c|}{0.206} & \multicolumn{2}{|c|}{0.800} & \multicolumn{4}{|c|}{0.036} & \\
\hline
\end{tabular}

2.3. Statistical Analysis. Demographic and clinical characteristics were collected and included gender, age, place of residence, degree of education, neurological deficit, pain, radiculopathy, systemic symptoms, neurological manifestations, imaging features, pathologic vertebrae, treatment options, posttreatment complications, $\mathrm{Hb}$, and ESR. All statistical analyses were performed using SPSS statistical software version 26.0 (IBM, Chicago, USA). The chisquared $\left(\chi^{2}\right)$ test was used to analyze the collected data, with $p<0.05$ considered statistically significant.

\section{Results}

3.1. General Information. A total of 556 eligible cases of confirmed STB were reviewed in this study (Table 1). Among them, there were $361(64.93 \%)$ patients between the ages of 18 and 45 years and 98 (17.63\%) patients between 46 and 60 years. There was a statistically significant difference between the age distribution of patients $(p=0.003)$ during the reviewed period. During this 9 -year period, the incidence of STB was relatively stable (30-112 new cases per year), and the gender proportions, male $(50.17 \%)$ and females $(49.83 \%)$, remained virtually unchanged, without any statistically significant differences $(p>0.05)$. Among the cases reviewed, rural residents accounted for $63.49 \%$, while urban residents accounted for $36.51 \%$, but the difference was not statistically significant $(p>0.05)$. In terms of occupational distribution, there were 338 farmers (60.79\%), 89 bluecollar workers (16.01\%), 26 students (4.68\%), and 103 $(18.53 \%)$ in other forms of occupation. The result of our correlation analysis between occupation and years was statistically significant $(p=0.036)$. The average interval between onset of symptoms and hospitalization was 18.0 months (range: 1 day-220 months). The average intervals for rural patients were significantly longer (25.5 months) compared to urban patients (9.1 months) $(p<0.05)$.

There is regional and local distribution of patients in the major cities of Guangxi Province. During the period studied,
Nanning City had the highest incidence of STB (17.09\%), followed by Guigang 15.83\%, Chongzuo 12.95\%, Wuzhou $9.17 \%$, Yulin 6.47\%, Laibin 6.29\%, Baise 6.29\%, Guilin $5.94 \%$, etc. Figures 1(a) and 1(b).

3.2. Clinical Data Analysis. The clinical manifestations of the STB patients involved in this study are summarized in Table 2. Lower back pain was reported in $78.60 \%$ of patients, followed by radicular pain (51.98\%) and systemic toxemic symptoms $(43.53 \%)$. The results of our correlation analysis revealed no correlation between lower back pain, systemic poisoning, and annual incidence (years) during the period of the study $(p>0.05)$. However, there was a statistically significant correlation between radicular pain and annual TB incidence $(p<0.05)$.

According to the Frankel classification, there were 15 (2.7\%) grade A, 25 (4.5\%) grade B, 56 (10.07\%) grade C, $204(36.69 \%)$ grade D, and 256 grade $\mathrm{E}(46.04 \%)$ cases (Table 2). However, there was no statistically significant difference between the Frankel grades and annual disease incidence $(p>0.05)$. During follow-up examination, 379 patients $(68.17 \%)$ of the 556 reviewed cases reported vertebral tenderness during physical examination; also, 367 patients $(66.01 \%)$ reported vertebral percussion pain. In 153 patients (27.52\%), kyphosis deformities were observed during physical examination.

3.3. Radiographic and Laboratory Examinations. The summary of the analysis of the radiographic findings is shown in Table 3. The most common pathologic lesion locations were lumbar spine $(43.53 \%)$, thoracic spine $(32.55 \%)$, thoracolumbar spine $(6.65 \%)$, cervicothoracic spine $(6.29 \%)$, sacral spine (4.14\%), lumbosacral spine (3.96\%), and cervical spine $(2.88 \%)$. There was no statistically significant difference in pathological location and annual incidence $(p>0.05)$. Among the cases reviewed, $72.66 \%$ involved 2 vertebral segments, while $16.73 \%$ involved 3 or more segments. Abscesses were detected using CT/MRI, with 349 of 


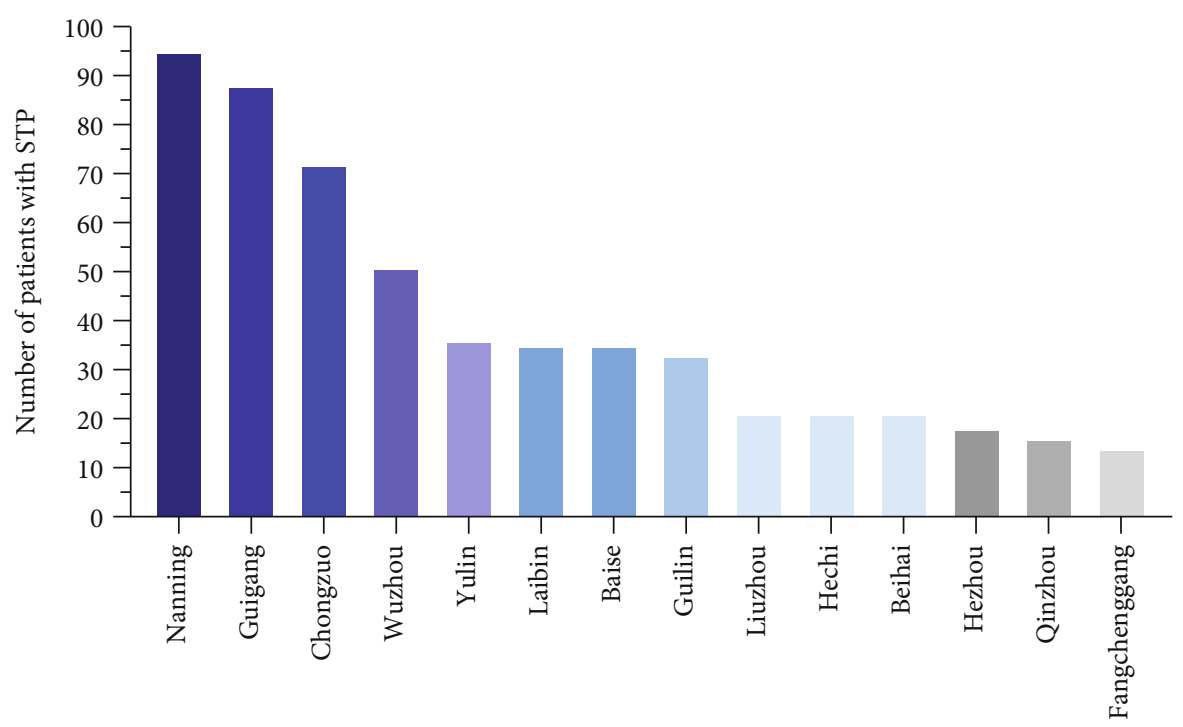

(a)

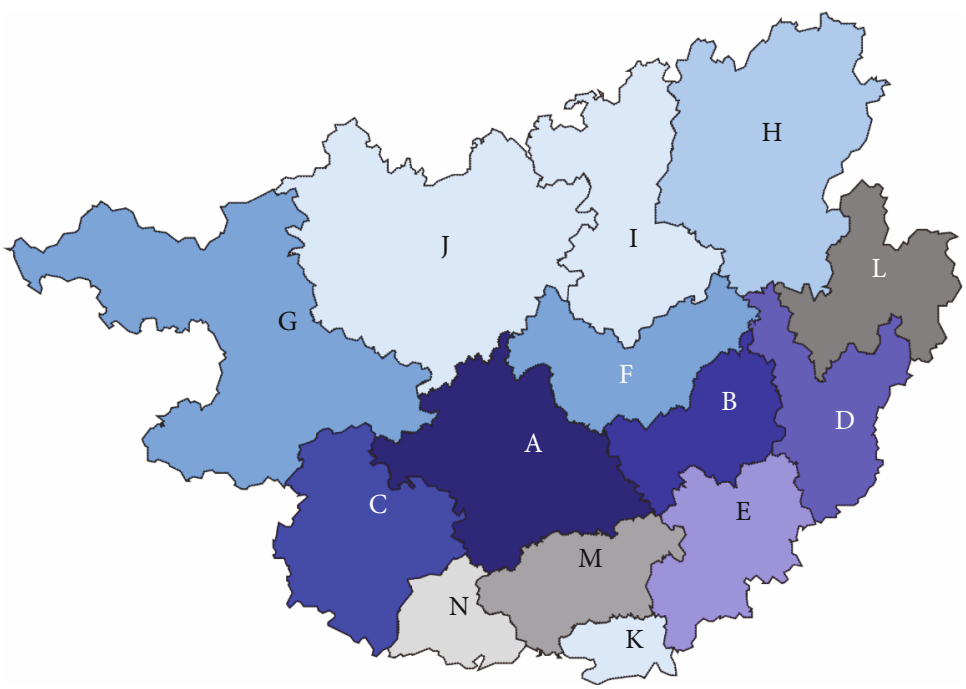

(b)

FIgURE 1: The geographical location and the number of patients in the main cities of Guangxi. (a) The number of STB patients in major cities. Nanning 17.09\%, Guigang 15.83\%, Chongzuo 12.95\%, Wuzhou 9.17\%, Yulin 6.47\%, Laibin 6.29\%, Baise 6.29\%, Guilin 5.94\%, Liuzhou 3.78\%, Hechi 3.78\%, Beihai 3.78\%, Hezhou 3.24\%, Qinzhou 2.88\%, and Fangchenggang 2.51\%. (b) The geographical location of major cities: Nanning (A), Guigang (B), Chongzuo (C), Wuzhou (D), Yulin (E), Laibin (F), Baise (G), Guilin (H), Liuzhou (I), Hechi (J), Beihai (K), Hezhou (L), Qinzhou (M), and Fangchenggang (N).

the 556 patients $(62.77 \%)$ diagnosed with paravertebral, prevertebral, or psoas major abscesses.

The normal range of hemoglobin is $115-150 \mathrm{~g} / \mathrm{L}$. In this study, patients' hemoglobin levels ranged between 61 and $164 \mathrm{~g} / \mathrm{L}$. Among the cases reviewed, 50.76\% had $\mathrm{Hb}$ ranging between 90 and $115 \mathrm{~g} / \mathrm{L} ; 3.12 \%$ had $\mathrm{Hb}$ ranging between 80 and $90 \mathrm{~g} / \mathrm{L}$, while $1.03 \%$ were below $80 \mathrm{~g} / \mathrm{L}$. Patient ESR ranged between 2 and $152 \mathrm{~mm} / \mathrm{h}$. In 109 patients, it was below $20 \mathrm{~mm} / \mathrm{h}$, while it ranged between 20 and 100 in 301 patients, with 146 patients having ESR higher than $100 \mathrm{~mm} / \mathrm{h}$. The range for CRP in this study was $0.11-204.31 \mathrm{mg} / \mathrm{L}$. Among the cases reviewed, there were 127 patients $(22.84 \%)$ with normal CRP, while the rest had varying degrees of CRP elevation, with the highest being $204.31 \mathrm{mg} / \mathrm{L}$.
3.4. Treatment and Prognosis. The treatment options administered are summarized in Table 4. In 504 (90.65\%) of patients, preoperative antituberculosis chemotherapy was used prior to surgical treatment and achieved good efficacy (Figure 2), while 52 (9.35\%) patients were treated with only antituberculosis chemotherapy. During the 9-year period, patients who underwent chemotherapy and surgery were greater than those treated with only chemotherapy; however, there was no statistically significant difference between them $(p>0.05)$. The primary surgical methods were anterior debridement, bone grafting, and internal fixation; anteriorlateral debridement, bone grafting, and internal fixation; and posterior debridement, bone grafting, and internal fixation. 
TABLE 2: Clinical manifestations of patients with STB.

\begin{tabular}{|c|c|c|c|c|c|c|c|c|c|c|c|c|}
\hline \multirow{3}{*}{ Year } & \multicolumn{6}{|c|}{ Clinical presentation } & \multirow{2}{*}{\multicolumn{5}{|c|}{ Neurological injury (Frankel classification) }} & \multirow{3}{*}{ Total } \\
\hline & \multicolumn{2}{|c|}{ Back pain } & \multicolumn{2}{|c|}{ Radiating pain } & \multicolumn{2}{|c|}{$\begin{array}{c}\text { Systemic } \\
\text { toxicity } \\
\text { symptoms }\end{array}$} & & & & & & \\
\hline & Yes & No & Yes & No & Yes & No & A & $\mathrm{B}$ & $\mathrm{C}$ & $\mathrm{D}$ & $\mathrm{E}$ & \\
\hline 2011 & 45 & 15 & 34 & 26 & 25 & 35 & 2 & 1 & 4 & 20 & 33 & 60 \\
\hline 2012 & 31 & 9 & 26 & 14 & 19 & 21 & 1 & 3 & 5 & 13 & 18 & 40 \\
\hline 2013 & 29 & 10 & 17 & 12 & 16 & 23 & 3 & 4 & 4 & 12 & 16 & 39 \\
\hline 2014 & 19 & 11 & 15 & 15 & 17 & 13 & 1 & 2 & 5 & 9 & 13 & 30 \\
\hline 2015 & 31 & 7 & 13 & 25 & 13 & 25 & 1 & 1 & 4 & 15 & 17 & 38 \\
\hline 2016 & 63 & 13 & 54 & 22 & 30 & 46 & 1 & 2 & 6 & 22 & 45 & 76 \\
\hline 2017 & 93 & 19 & 53 & 59 & 59 & 53 & 3 & 5 & 12 & 42 & 50 & 112 \\
\hline 2018 & 65 & 20 & 42 & 43 & 38 & 47 & 1 & 3 & 7 & 39 & 35 & 85 \\
\hline 2019 & 61 & 15 & 35 & 41 & 25 & 51 & 2 & 4 & 9 & 32 & 29 & 76 \\
\hline Total & 437 & 119 & 289 & 257 & 242 & 314 & 15 & 25 & 56 & 204 & 256 & 556 \\
\hline Rate (\%) & 78.60 & 21.40 & 51.98 & 48.02 & 43.53 & 56.47 & 2.70 & 4.50 & 10.07 & 36.69 & 46.04 & \\
\hline$\chi^{2}$ & & & & & & & & & 23.44 & & & \\
\hline$p$ & & & & & & & & & 0.864 & & & \\
\hline
\end{tabular}

TABLE 3: Imaging findings of patients with STB.

\begin{tabular}{|c|c|c|c|c|c|c|c|c|c|c|c|c|c|}
\hline \multirow{2}{*}{ Year } & \multicolumn{7}{|c|}{ Lesion sites } & \multicolumn{3}{|c|}{$\begin{array}{c}\text { Involved number of } \\
\text { vertebrae }\end{array}$} & \multicolumn{2}{|c|}{ Abscess } & \multirow{2}{*}{ Tota } \\
\hline & $\mathrm{C}$ & $\mathrm{CT}$ & $\mathrm{T}$ & $\mathrm{TL}$ & $\mathrm{L}$ & LS & S & 1 & 2 & $\geq 3$ & Yes & No & \\
\hline 2011 & 2 & 8 & 18 & 5 & 21 & 2 & 4 & 7 & 41 & 12 & 39 & 21 & 60 \\
\hline 2012 & 2 & 4 & 10 & 4 & 15 & 2 & 3 & 6 & 25 & 9 & 23 & 17 & 40 \\
\hline 2013 & 1 & 2 & 10 & 4 & 17 & 2 & 3 & 4 & 23 & 12 & 26 & 13 & 39 \\
\hline 2014 & 1 & 1 & 8 & 3 & 14 & 2 & 1 & 5 & 21 & 4 & 21 & 9 & 30 \\
\hline 2015 & 2 & 3 & 12 & 3 & 14 & 2 & 2 & 6 & 27 & 5 & 24 & 14 & 38 \\
\hline 2016 & 1 & 2 & 27 & 4 & 37 & 2 & 3 & 7 & 57 & 12 & 48 & 28 & 76 \\
\hline 2017 & 3 & 8 & 39 & 7 & 49 & 4 & 2 & 8 & 92 & 12 & 76 & 36 & 112 \\
\hline 2018 & 2 & 3 & 30 & 4 & 42 & 2 & 2 & 9 & 65 & 11 & 49 & 36 & 85 \\
\hline 2019 & 2 & 4 & 27 & 3 & 33 & 4 & 3 & 7 & 53 & 16 & 43 & 33 & 76 \\
\hline Total & 16 & 35 & 181 & 37 & 242 & 22 & 23 & 59 & 404 & 93 & 349 & 207 & 556 \\
\hline Rate (\%) & 2.88 & 6.29 & 32.55 & 6.65 & 43.53 & 3.96 & 4.14 & 10.61 & 72.66 & 16.73 & 62.77 & 37.23 & \\
\hline$\chi^{2}$ & & & & 27.850 & & & & & 17.354 & & & & \\
\hline$p$ & & & & 0.991 & & & & & 0.363 & & & & \\
\hline
\end{tabular}

C: cervical; CT: cervicothoracic; T: thoracic; TL: thoracolumbar; L: lumbar; LS: lumbosacral; S: sacrococcygeal.

The summary of patients' prognoses is shown in Table 4. A total of 486 patients $(78.41 \%)$ were classified as "cured" after receiving treatment. Local recurrence was observed in 21 patients $(3.78 \%)$. Such patients received continued antituberculosis chemotherapy and underwent revision surgery. $49(8.81 \%)$ patients were classified as "other" because of incomplete follow-up data, loss of contact, failure to heal, or transferred treatment. No patient died during the entire follow-up period.

3.5. Comorbidities. Among the 556 patients reviewed, 226 (40.65\%) had PTB, 85 (15.29\%) had hepatitis B, 74
(13.30\%) had diabetes, and 44 (7.91\%) had hypertension (Figures 3(a)-3(d)).

Among the patients with pulmonary tuberculosis (Table 5), 64.60\% were males and $35.4 \%$ were females. Moreover, $40.71 \%$ of them reported symptoms of systemic toxemia, $69.91 \%$ had paravertebral abscesses, and $75.66 \%$ had neurological impairments. Surgical treatment was performed in $86.73 \%$ of the pulmonary tuberculosis patients, of which $82.30 \%$ were classified as "cured" after the treatment. The results of our correlation analysis revealed that there were statistically significant differences between gender, paravertebral abscess, nerve injury, surgery, and cure 
TABLE 4: Treatment and prognosis of patients with STB.

\begin{tabular}{|c|c|c|c|c|c|c|}
\hline \multirow{2}{*}{ Year } & \multicolumn{2}{|c|}{ Treatment } & \multicolumn{3}{|c|}{ Prognosis } & \multirow{2}{*}{ Total } \\
\hline & Only anti-TB chemotherapy & Anti-TB chemotherapy and surgery & Cured & Relapse & Others & \\
\hline 2011 & 6 & 54 & 56 & 3 & 1 & 60 \\
\hline 2012 & 5 & 35 & 35 & 2 & 3 & 40 \\
\hline 2013 & 7 & 32 & 34 & 3 & 2 & 39 \\
\hline 2014 & 3 & 27 & 25 & 1 & 4 & 30 \\
\hline 2015 & 4 & 34 & 37 & 0 & 1 & 38 \\
\hline 2016 & 6 & 70 & 62 & 4 & 10 & 76 \\
\hline 2017 & 11 & 101 & 98 & 3 & 11 & 112 \\
\hline 2018 & 6 & 79 & 75 & 3 & 8 & 85 \\
\hline 2019 & 4 & 72 & 64 & 2 & 9 & 76 \\
\hline Total & 52 & 504 & 486 & 21 & 49 & 556 \\
\hline Rate (\%) & 9.35 & 90.65 & 78.41 & 3.78 & 8.81 & \\
\hline$\chi^{2}$ & & 5.835 & & 17.847 & & \\
\hline$p$ & & 0.666 & & 0.333 & & \\
\hline
\end{tabular}

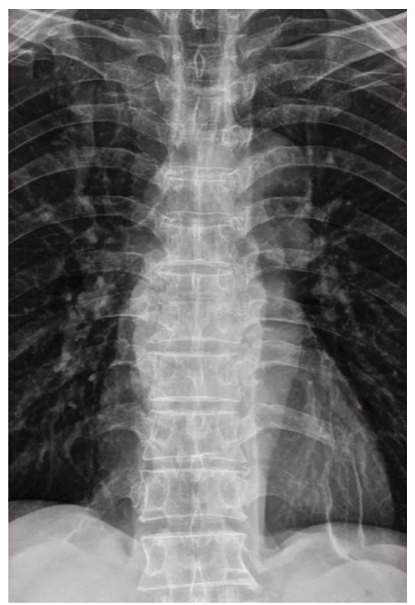

(a)

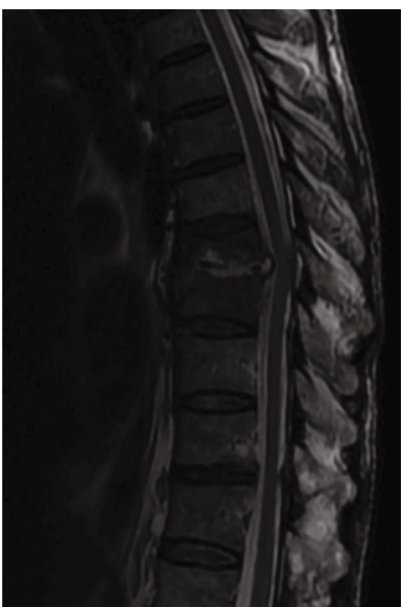

(e)

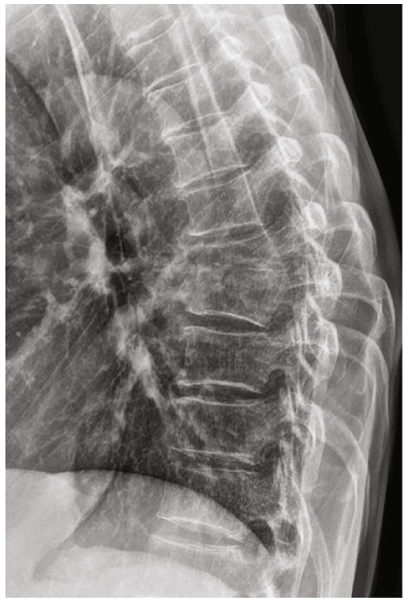

(b)

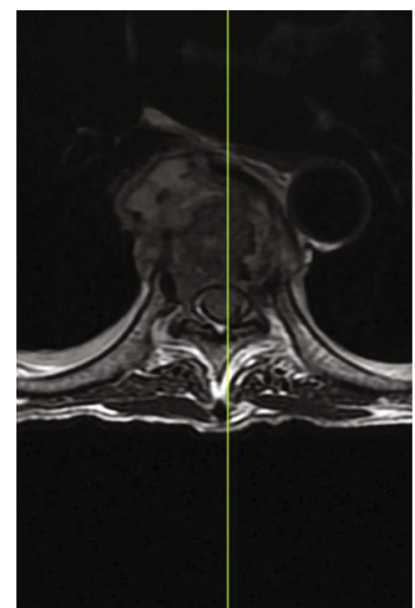

(f)

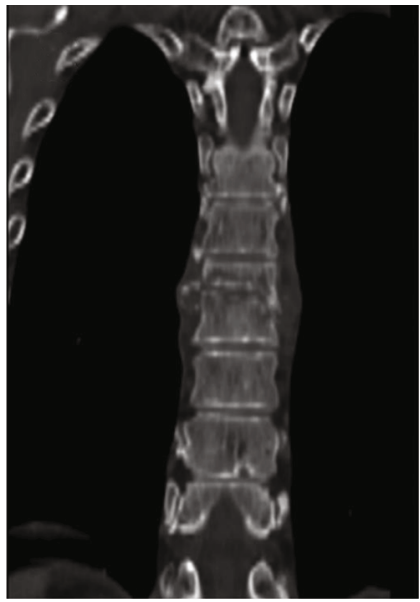

(c)

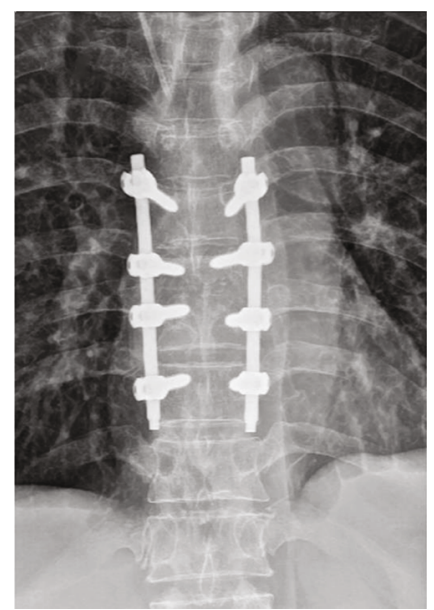

(g)

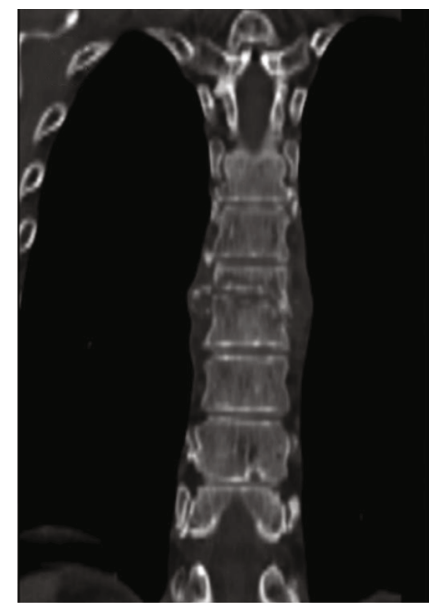

(d)

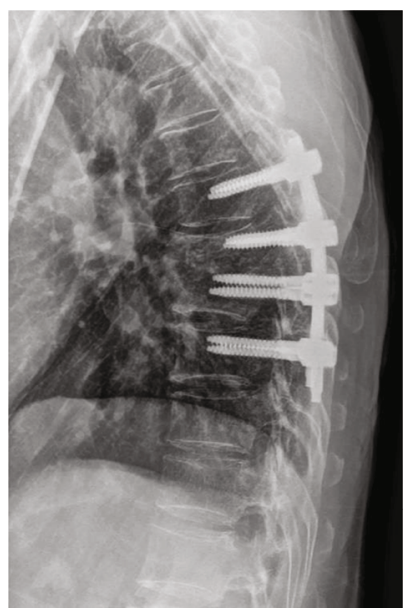

(h)

Figure 2: A 68-year-old male with T5/6 lesions. (a-f) The preoperative imaging data showed T5/6 vertebral bodies' destructions with kyphosis deformity and spinal cord severely compressed. The postoperative sagittal and coronal X-ray (g, h) indicated that the kyphosis got obviously improved. 


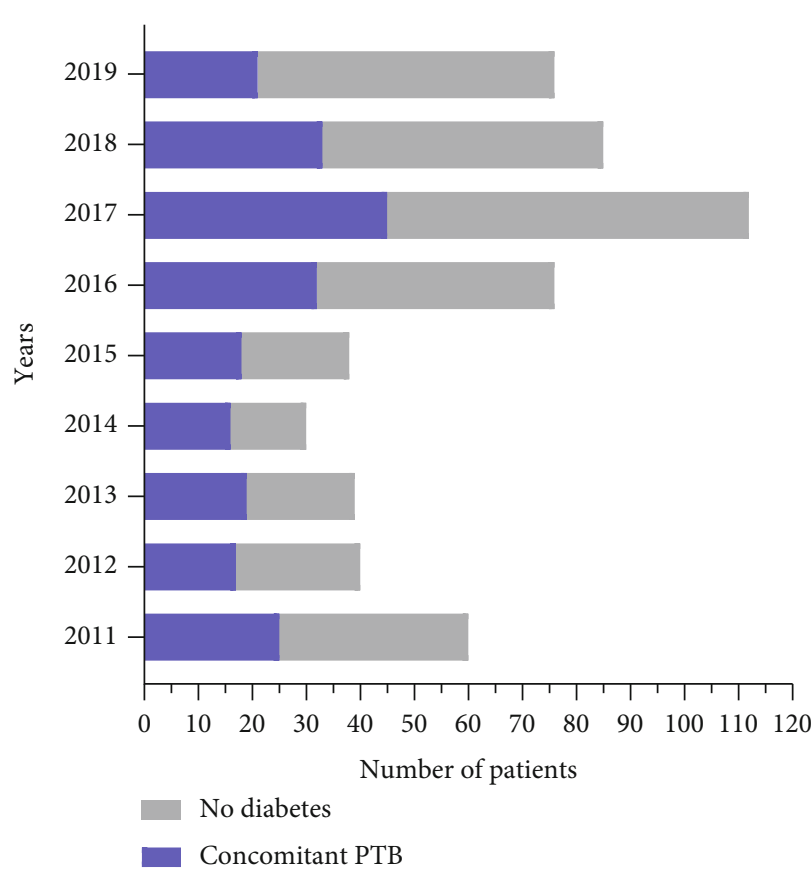

(a)

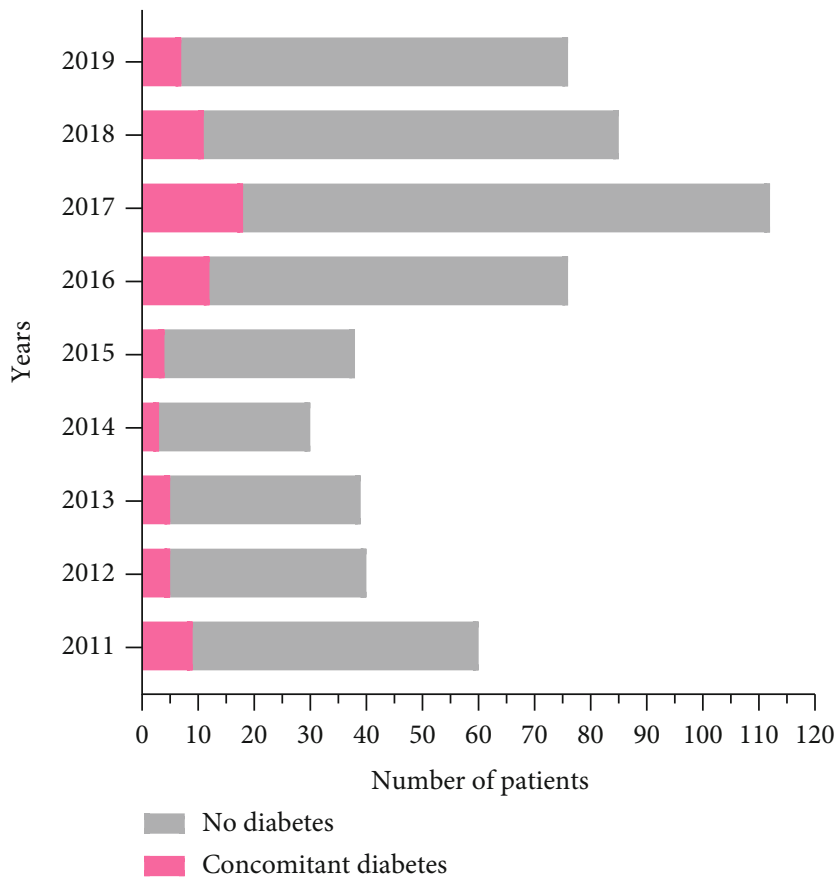

(c)

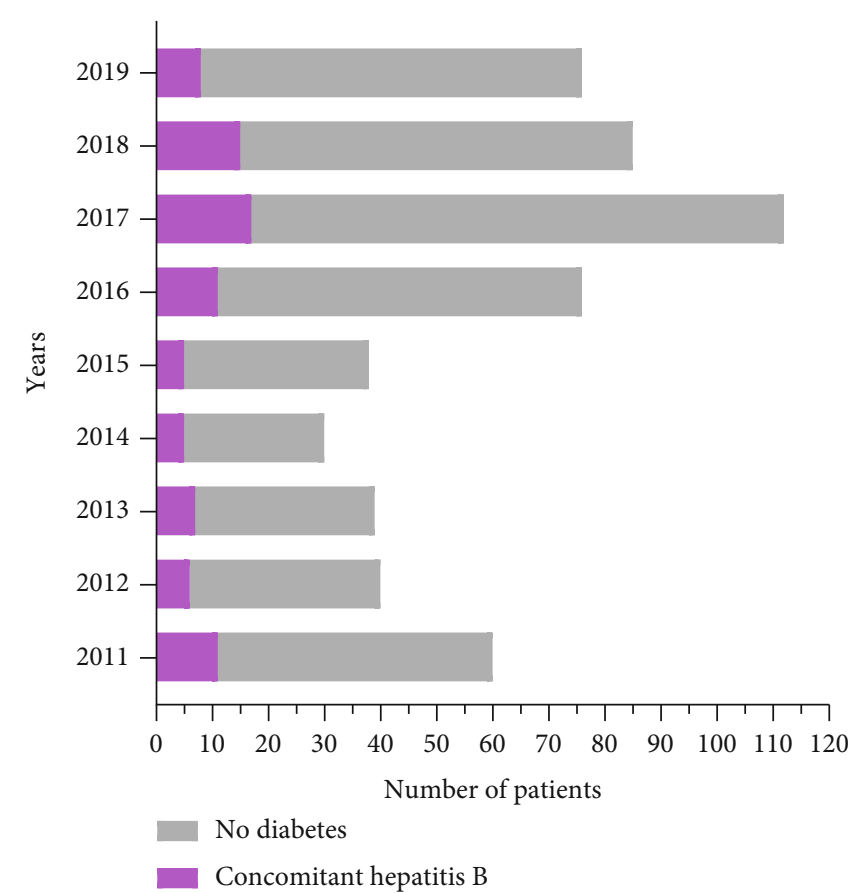

(b)

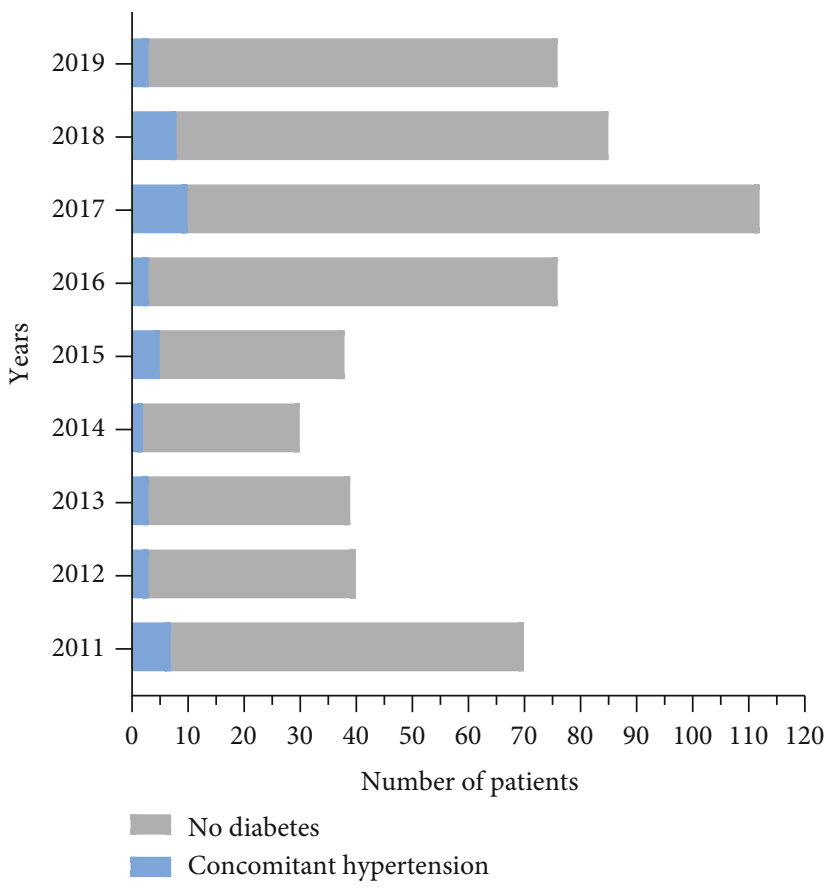

(d)

FIgURE 3: Comorbidities. (a) The number of patients with concomitant PTB. (b) The number of patients with concomitant hepatitis B. (c) The number of patients with concomitant diabetes. (d) The number of patients with concomitant hypertension.

rate in patients with pulmonary TB diagnosis $(p<0.05)$. On the contrary, there was no statistically significant correlation between symptoms of systemic toxemia and pulmonary tuberculosis diagnosis $(p>0.05)$.

Among the patients reviewed, $48.64 \%$ had diabetes (Table 6). Among which, patients 45 years old and over accounted for $82.43 \%$, with $43.24 \%$ of them reporting systemic toxemic symptoms. Moreover, neurological manifes- tations were reported in $52.70 \%$, while paravertebral abscesses were observed in $71.62 \%$. Following surgical treatment, $78.37 \%$ of the diabetic patients were classified as "cured." Age, surgical treatment, and cure rate were found to be correlated with diabetes $(p<0.05)$. However, systemic toxemia, paravertebral abscesses, and neurological impairments were not significantly correlated with diabetes $(p>0.05)$. 
TABLE 5: Correlation analysis between STB and PTB.

\begin{tabular}{|c|c|c|c|c|c|c|c|c|}
\hline \multirow{2}{*}{ Variate } & \multirow{2}{*}{ Type } & \multicolumn{2}{|c|}{ With PTB } & \multicolumn{2}{|c|}{ Without PTB } & \multirow{2}{*}{ Total } & \multirow{2}{*}{$\chi^{2}$} & \multirow{2}{*}{$p$} \\
\hline & & $N$ & $\%$ & $N$ & $\%$ & & & \\
\hline \multirow{2}{*}{ Gender } & Male & 146 & 64.60 & 133 & 40.30 & 279 & \multirow{2}{*}{31.680} & \multirow{2}{*}{$<0.001$} \\
\hline & Female & 80 & 35.4 & 197 & 59.7 & 277 & & \\
\hline \multirow{2}{*}{ Systemic toxicity symptoms } & Yes & 91 & 40.71 & 151 & 45.76 & 242 & \multirow{2}{*}{1.646} & \multirow{2}{*}{0.200} \\
\hline & No & 135 & 59.29 & 179 & 54.24 & 314 & & \\
\hline \multirow{2}{*}{ Abscess } & Yes & 158 & 69.91 & 131 & 39.70 & 349 & \multirow{2}{*}{49.059} & \multirow{2}{*}{$<0.00$} \\
\hline & No & 68 & 30.09 & 199 & 60.3 & 207 & & \\
\hline \multirow{2}{*}{ Neurological injury } & Yes & 171 & 75.66 & 129 & 39.09 & 300 & \multirow{2}{*}{72.219} & \multirow{2}{*}{$<0.00$} \\
\hline & No & 55 & 24.34 & 201 & 60.91 & 256 & & \\
\hline \multirow{2}{*}{ Surgery } & Yes & 196 & 86.73 & 308 & 93.33 & 504 & \multirow{2}{*}{6.908} & \multirow{2}{*}{0.009} \\
\hline & No & 30 & 13.27 & 22 & 6.67 & 52 & & \\
\hline \multirow{2}{*}{ Prognosis } & Cure & 186 & 82.30 & 300 & 90.90 & 486 & \multirow{2}{*}{9.032} & \multirow{2}{*}{0.003} \\
\hline & Other & 40 & 17.7 & 30 & 9.1 & 70 & & \\
\hline
\end{tabular}

TABLE 6: Correlation analysis between STB and diabetes.

\begin{tabular}{|c|c|c|c|c|c|c|c|c|}
\hline \multirow{2}{*}{ Variate } & \multirow{2}{*}{ Type } & \multicolumn{2}{|c|}{ With diabetes } & \multicolumn{2}{|c|}{ Without diabetes } & \multirow{2}{*}{ Total } & \multirow{2}{*}{$\chi^{2}$} & \multirow{2}{*}{$p$} \\
\hline & & $N$ & $\%$ & $N$ & $\%$ & & & \\
\hline \multirow{2}{*}{ Age (years) } & $>45$ & 61 & 82.43 & 95 & 19.71 & 156 & \multirow{2}{*}{125.032} & \multirow{2}{*}{$<0.001$} \\
\hline & $<45$ & 13 & 17.57 & 387 & 80.29 & 400 & & \\
\hline \multirow{2}{*}{ Systemic toxicity symptoms } & Yes & 32 & 43.24 & 210 & 43.56 & 242 & \multirow{2}{*}{0.003} & \multirow{2}{*}{0.958} \\
\hline & No & 42 & 56.76 & 272 & 56.44 & 314 & & \\
\hline \multirow{2}{*}{ Abscess } & Yes & 53 & 71.62 & 296 & 61.41 & 349 & \multirow{2}{*}{2.862} & \multirow{2}{*}{0.091} \\
\hline & No & 21 & 28.38 & 186 & 38.59 & 207 & & \\
\hline \multirow{2}{*}{ Neurological injury } & Yes & 39 & 52.70 & 261 & 54.15 & 300 & \multirow{2}{*}{0.054} & \multirow{2}{*}{0.816} \\
\hline & No & 35 & 47.3 & 221 & 45.85 & 256 & & \\
\hline \multirow{2}{*}{ Surgery } & Yes & 36 & 48.64 & 468 & 97.1 & 504 & \multirow{2}{*}{177.603} & \multirow{2}{*}{$<0.001$} \\
\hline & No & 38 & 51.36 & 14 & 2.90 & 52 & & \\
\hline \multirow{2}{*}{ Prognosis } & Cure & 58 & 78.37 & 428 & 88.79 & 486 & \multirow{2}{*}{6.327} & \multirow{2}{*}{0.012} \\
\hline & Other & 16 & 21.63 & 54 & 11.21 & 70 & & \\
\hline
\end{tabular}

\section{Discussion}

Tuberculosis continues to threaten public health, especially in developing countries. Spinal tuberculosis (Pott disease) was first described in 1782 by Percival Pott [9]. In developing countries, spinal tuberculosis mostly occurs among young and middle-aged people, while in the developed world, the elderly are the most affected $[10,11]$. An epidemiological survey of spinal tuberculosis in the United States between 2002 and 2011 revealed an average age of 51 years (35-65 years), with $24.5 \%$ of cases over the age of 65 years [12]. In this study, $64.93 \%$ of the patients were between the ages of 18 and 45 years, while $17.63 \%$ were between 46 and 60 years old. This is possible because patients in the age group of 18-60 years perform physical labor for a long time, which increases the load on the spine, which leads to chronic spinal injury, which can easily lead to infection of Mycobacterium tuberculosis. In this study, patients younger than 18 years accounted for only $7.01 \%$, which may be explained by the fact that the protective effect of BCG vaccination con- tinues into the adolescence stage [13]. According to a 2020 report by the World Health Organization [2], the male to female ratio of newly diagnosed tuberculosis in China was 2.19:1. However, in this study, the male to female ratio was $1: 1$, with no statistically significant change in the 9year period of the study. In Guizhou, which is also an underdeveloped southwestern region of China, the ratio of male to female in STB is $1.1: 1$ [14]. This similarity in the sex ratio may be due to the similarities in the economic, medical, and educational levels of the two regions. In our study, the number of STB patients after 2016 has an increasing trend than before, but it is not statistically significant. This may be because in recent years, the country has strengthened poverty alleviation and the country has increased its medical insurance investment. The awareness of hospital visits has increased, so the number of visits has increased.

It is reported that $80 \%$ of STB patients worldwide are in poor and developing countries. Studies have also reported that $80 \%$ of STB patients in China are from rural areas [15]; however, in this study, the ratio was slightly lower at 
$63.48 \% .60 .79 \%$ of patients were farmers. The average interval between the onset of symptoms and hospitalization of rural patients was 25.5 months; on the contrary, that for the patient from urban areas was 9.1 months. This may be related to the low level of health awareness, education, and the overall level of socioeconomic development in Guangxi Province. According to the urban distribution of STB, Nanning City, Guigang City, and Chongzuo City have more STB patients than other major cities in China. These differences can be attributed to the lower standard of medical care and socioeconomic development in these cities.

Unlike pulmonary tuberculosis, the most common manifestation of spinal tuberculosis is lower back pain [16]. Other major symptoms include systemic toxemic symptoms such as low-grade fever, night sweats, and weight loss. In this study, $78.60 \%$ of patients reported lower back pain, while $43.53 \%$ reported systemic toxemic symptoms. The main reason why STB patients seek medical evaluation and treatment is lower back pain. In this study, vertebral tenderness was reported in $68.17 \%$, while vertebral percussion pain was reported in $66.01 \%$ of patients during physical examination. Kyphosis was also observed in $27.52 \%$ during the physical. The contiguous bone necrosis associated with tuberculosis coupled with psoas, paravertebral, or prevertebral abscess formation may result in neurological manifestations such as back pain and radiating pain. Studies have reported that delayed treatment of STB could result in neurological damage and even paralysis [17]. In this study, 51.98\% of patients reported neuropathic pain. Varying degrees of neurological impairment were observed in $53.96 \%$ of cases. Severe neurological impairments were observed in $7.2 \%$ of cases $(2.70 \%$ Frankel grade A; $4.50 \%$ grade B). Following surgical treatment, varying degrees of neurological function recovery were observed in such patients, confirming the poorer recovery rate among patients with severe neurological impairment on admission.

According to the available literature, the most common site for spinal tuberculosis is the lumbar and thoracic spine [18]. In this study, lumbar tuberculous lesions accounted for $43.53 \%$, while thoracic and thoracolumbar lesions accounted for $32.55 \%$ and $6.65 \%$, respectively. This localization may be related to the anatomical factors of the high level of mobility and load forces in the lumbar region in the thoracic region. STB lesions usually involve two or more vertebrae. This is because the segmental arteries bifurcate to supply two adjacent vertebrae, making the hematogenous spread to adjacent vertebrae easy. MTB involvement of the anterior or posterior longitudinal ligament may also enable multiple adjacent vertebrae involvement [8]. In this study, $72.66 \%$ of cases involved 2 adjacent vertebrae, while $16.73 \%$ involved 3 or more vertebrae, while paravertebral abscesses were found in $62.77 \%$ of patients.

The hemoglobin level has been used as an indirect indicator of health and nutritional status, and studies have reported that tuberculosis causes weight loss and malnutrition [19]. Thus, in this study, we analyzed the hemoglobin levels to determine the nutritional status of patients. In this study, anemia was observed in $54.91 \%$ of patients, thus con- firming the high incidence of malnutrition in tuberculosis patients. Thus, our results also revealed that more attention should be paid to nutritional supplementation before, during, and after antituberculosis chemotherapy or surgical treatment for TB-related diseases. ESR and CRP, which are used as indirect indicators of inflammation and inflammatory reactions, have high sensitivity but low specificity. However, they can still be used to monitor disease activity during follow-up. The average ESR is $43.5 \mathrm{~mm} / \mathrm{h}$ [20]; however, in this study, ESR ranged between 2 and $152 \mathrm{~mm} / \mathrm{h}$. The average CRP in STB patients has been reported to range between 6 and $197 \mathrm{mg} / \mathrm{L}$ [21]. However, in this study, it ranged between 0.11 and $204.31 \mathrm{mg} / \mathrm{L}$. Thus, it confirms that inflammatory reactions are common in tuberculosis patients and that ESR and CRP can be used as indirect indicators of postoperative prognosis.

Antituberculosis drugs are currently the basis for spinal tuberculosis treatment. Surgical treatment on the other hand plays an important adjuvant role. This is because surgical risks such as loss of life, infection, metastasis, and postoperative recurrence are significantly increased in the absence of preoperative chemotherapy [22]. The American Thoracic Society recommends 6-9 months of chemotherapy for STB, and many studies have demonstrated the effectiveness of such a program [23]. Surgical indications for STB include spinal cord and nerve root compression or injury, severe kyphotic deformity or progressive kyphosis, spinal column instability, and abscesses. Surgical decompression is recommended as soon as possible following effective chemotherapy; this not only promotes recovery of nerve function and spinal column stability but also provides specimens for confirmatory pathological examination of surgical samples to help guide chemotherapy. In this study, $90.65 \%$ of patients received preoperative antituberculosis, with a cure rate of $78.41 \%$. The recurrence rate was 3.78\% (21 patients). Reasons for relapse may include unstable antituberculosis drug treatment, malnutrition, comorbidities, and lack of rest. Relapsed patients were cured by revision surgery, including debridement, drainage, and local catheter infusion of antituberculosis drugs.

Studies have reported that between $33 \%$ and $50 \%$ of STB patients have a history of pulmonary tuberculosis [24, 25]. In this study, $40.65 \%$ of the STB patients reviewed had PTB history, the majority of them being males, who were also at risk of developing paravertebral abscess lesions and nerve damage. This could be attributed to the high incidence of smoking and drinking among Chinese men. Furthermore, the majority of patients reviewed in this study were farmers or had blue-collar jobs, where manual labor is the norm.

The immunosuppression observed in diabetes patients has been reported to result in increased susceptibility to infections and poor tissue repair abilities [26]. Active tuberculosis is an infection that can aggravate diabetes and may even induce acute ketoacidosis. Diabetes and tuberculosis patients usually have the characteristics of the long course, severe illness, rapid progress, slow recovery, etc., which are difficult to treat $[27,28]$. In addition, patients with diabetes and STB have impaired anti-infection and tissue repair capabilities, which affect the prognosis and sometimes lead to 
surgical failure. In this study, the majority of STB patients over 45 years with diabetes were treated conservatively unless necessary.

This study has several limitations. Firstly, it is a retrospective study of 556 inpatients in 2 tertiary hospitals. Thus, the sample size is relatively small. Secondly, this study did not include outpatients, which may lead to a high proportion of surgically treated patients and no HIV/ARDS cases. Finally, this study did not obtain the results of antituberculosis drug resistance.

\section{Conclusion}

Spinal tuberculosis is still a serious problem that endangers public health. Lower back pain is the most common clinical manifestation of STB, and the lumbar vertebrae are the most commonly affected. More attention should be paid to rural residents, farmers, blue-collar workers, elderly, and diabetic patients who should be the main focus of spinal tuberculosis preventive measures, especially in underdeveloped areas. Our results provided valuable information on the epidemiological characteristics of spinal tuberculosis in Guangxi Province, China, thus helping to raise local, regional, and national attention to it.

\section{Data Availability}

The data used to support the findings of this study are included within the article.

\section{Conflicts of Interest}

The authors have no conflicts of interest to declare.

\section{Acknowledgments}

The authors would like to thank colleagues from the Department of Spine and Osteopathy Surgery of Guangxi Medical University First Affiliated Hospital for their support and collaboration. This research was supported by the National Natural Science Foundation of China (Grant No. 82160420), Clinical Climbing Program of the First Affiliated Hospital of Guangxi Medical University (Grant No. YYZS2020022), Natural Science Fund of Guangxi Medical University for Young Scientists (Grant No. GXMUYSF201701), and Natural Science Foundation of Guangxi Zhuang Autonomous Region (Grant No. 2018JJB140199).

\section{References}

[1] Y. Zhao, M. Li, and S. Yuan, "Analysis of transmission and control of tuberculosis in Mainland China, 2005-2016, based on the age-structure mathematical model," International Journal of Environmental Research and Public Health, vol. 14, no. 10, p. 1192, 2017.

[2] L. Cormican, R. Hammal, J. Messenger, and H. J. Milburn, "Current difficulties in the diagnosis and management of spinal tuberculosis," Postgraduate Medical Journal, vol. 82, pp. $46-51,2006$.
[3] R. Lunt, "Bone and joint tuberculosis," British Medical Journal, vol. 2, pp. 907-909, 1979.

[4] H. Zeng, X. Shen, C. Luo et al., "360-degree cervical spinal arthrodesis for treatment of pediatric cervical spinal tuberculosis with kyphosis," BMC Musculoskeletal Disorders, vol. 17, p. $175,2016$.

[5] L. J. Wang, H. Q. Zhang, M. X. Tang, Q. L. Gao, Z. H. Zhou, and X. H. Yin, "Comparison of three surgical approaches for thoracic spinal tuberculosis in adult: minimum 5-year follow up," Spine, vol. 42, pp. 808-817, 2017.

[6] WHO, "Global tuberculosis report," 2020, http://www.who .int/tb/publications/global_report/en/.

[7] M. Fukui, K. Chiba, M. Kawakami et al., "An outcome measure for patients with cervical myelopathy: Japanese Orthopaedic Association Cervical Myelopathy Evaluation Questionnaire (Joacmeq): part 1," Journal of Orthopaedic Science, vol. 12, no. 3, pp. 227-240, 2007.

[8] Z. Liu, J. Wang, G. Z. Chen et al., "Clinical characteristics of 1378 inpatients with spinal tuberculosis in general hospitals in south-Central China," BioMed Research International, vol. 2019, Article ID 9765253, 11 pages, 2019.

[9] G. J. Gorse, M. J. Pais, J. A. Kusske, and T. C. Cesario, "Tuberculous spondylitis. A report of six cases and a review of the literature," Medicine, vol. 62, pp. 178-193, 1983.

[10] S. P. Chandra, A. Singh, N. Goyal et al., "Analysis of changing paradigms of management in 179 patients with spinal tuberculosis over a 12-year period and proposal of a new management algorithm," World Neurosurgery, vol. 80, no. 1-2, pp. 190-203, 2013.

[11] C. Wibaux, M. Moafo-Tiatsop, I. Andrei et al., "Changes in the incidence and management of spinal tuberculosis in a French university hospital rheumatology department from 1966 to 2010," Joint, Bone, Spine, vol. 80, no. 5, pp. 516-519, 2013.

[12] R. De la Garza Ramos, C. R. Goodwin, N. Abu-Bonsrah et al., "The epidemiology of spinal tuberculosis in the United States: an analysis of 2002-2011 data," Journal of Neurosurgery. Spine, vol. 26, pp. 507-512, 2017.

[13] K. X. Mao, C. X. Zhen, L. H. Yan, Q. Dan, M. X. Wang, and C. Y. Zhang, "Protective effect of vaccination of bacille Calmette-Guerin on children," Chinese Journal of Contemporary Pediatrics, vol. 5, no. 4, pp. 325-327, 2003.

[14] P. Wang, W. Liao, G. Cao, Y. Jiang, J. Rao, and Y. Yang, "Characteristics and management of spinal tuberculosis in tuberculosis endemic area of Guizhou Province: a retrospective study of 597 patients in a teaching hospital," BioMed Research International, vol. 2020, Article ID 1468457, 8 pages, 2020.

[15] W. Yu, "Technical Guidance Group of the Fifth National TB Epidemiological Survey the Office of the Fifth National TB Epidemiological Survey. The fifth national tuberculosis epidemiological survey in 2010," Chinese Journal of Antituberculosis, vol. 34, no. 8, pp. 485-508, 2012.

[16] R. K. Garg and D. S. Somvanshi, "Spinal tuberculosis: a review," The Journal of Spinal Cord Medicine, vol. 34, pp. 440-454, 2011.

[17] R. F. McLain and C. Isada, "Spinal tuberculosis deserves a place on the radar screen," Cleveland Clinic Journal of Medicine, vol. 71, no. 537-9, pp. 43-49, 2004.

[18] Y. Tang, W. J. Wu, S. Yang et al., "Surgical treatment of thoracolumbar spinal tuberculosis-a multicentre, retrospective, case-control study," Orthopaedic surgery and research, vol. 14 , no. 1, p. 233, 2019. 
[19] S. Hayashi, M. Takeuchi, K. Hatsuda et al., "The impact of nutrition and glucose intolerance on the development of tuberculosis in Japan," The International Journal of Tuberculosis and Lung Disease, vol. 18, no. 1, pp. 84-88, 2014.

[20] Y. Yao, W. Song, K. Wang et al., "Features of 921 patients with spinal tuberculosis: a 16-year investigation of a general hospital in southwest China," Orthopedics, vol. 40, article e1017, 2017.

[21] D. Mulleman, S. Mammou, I. Griffoul, A. Avimadje, P. Goupille, and J. P. Valat, "Characteristics of patients with spinal tuberculosis in a French teaching hospital," Joint, Bone, Spine, vol. 73, pp. 424-427, 2006.

[22] S. M. Tuli, "Tuberculosis of the spine: a historical review," Clinical Orthopaedics and Related Research, vol. 460, pp. 2938, 2007.

[23] Y. Maeda, K. Izawa, T. Nabeshima, and K. Yonenobu, "Tuberculous spondylitis in elderly Japanese patients," Journal of Orthopaedic Science, vol. 13, pp. 16-20, 2008.

[24] P. Schirmer, C. A. Renault, and M. Holodniy, "Is spinal tuberculosis contagious?," International Journal of Infectious Diseases, vol. 14, pp. e659-e666, 2010.

[25] C. Y. Weng, C. Y. Chi, P. J. Shih et al., "Spinal tuberculosis in non-HIV-infected patients: 10 year experience of a medical center in central Taiwan," Journal of Microbiology, Immunology, and Infection, vol. 43, no. 6, pp. 464-469, 2010.

[26] Y. X. Wang, H. Q. Zhang, W. Liao et al., "One-stage posterior focus debridement, interbody graft using titanium mesh cages, posterior instrumentation and fusion in the surgical treatment of lumbo-sacral spinal tuberculosis in the aged," International Orthopaedics, vol. 40, no. 6, pp. 1117-1124, 2016.

[27] S. Chen, M. V. Anderson, W. K. Cheng, and M. D. Wongworawat, "Diabetes associated with increased surgical site infections in spinal arthrodesis," Clinical Orthopaedics and Related Research, vol. 467, no. 7, pp. 1670-1673, 2009.

[28] S. Lin, M. Shen, and Y. Sun, "Epidemiological characteristics of tuberculosis patients complicated with diabetes in Shanghai," Zhonghua jie he he hu xi za zhi= Zhonghua jiehe he huxi zazhi $=$ Chinese journal of tuberculosis and respiratory diseases, vol. 21, no. 8, pp. 504-506, 1998. 